\title{
Estimation of Power Losses Caused by Supraharmonics
}

\author{
A. Novitskiy ${ }^{*}$, S. Schlegel, D. Westermann \\ Ilmenau University of Technology, Ilmenau, Germany
}

\begin{abstract}
Nowadays, the number of power electronic devices in electrical distribution networks rapidly increases. Modern generation and consumption units use high switching frequency power converters for the network connection and therefore cause voltage and current distortion at frequencies higher than $2 \mathrm{kHz}$ (so-called supraharmonics) in addition to conventional harmonics. Supraharmonics cause additional power losses in electrical equipment. The objective of this paper is to estimate the power losses caused by supraharmonics. The focus is on the method of assessing the supraharmonic power losses, which combines the analytic determination of AC cable resistances at supraharmonic frequencies and the use of supraharmonic currents measured in a real $\mathrm{MV} / \mathrm{LV}$ cable network. The paper shows that the supraharmonic power losses in an MV cable can reach the values of several percent of power losses at the fundamental frequency and can exceed ten percent of power losses caused by conventional current harmonics.
\end{abstract}

Index Terms: Power losses, power quality, supraharmonics.

\section{INTRODUCTION}

The increase in the number of power electronic devices in electrical distribution networks has been a characteristic trend in the development of electric power supply for many years. Modern generation and consumption units use high switching frequency power converters for the network connection, which leads to voltage and current distortion at frequencies higher than $2 \mathrm{kHz}$ (so-called supraharmonics) in addition to conventional harmonics.

\footnotetext{
${ }^{*}$ Corresponding author.

E-mail: Alexander.Novitskiy@TU-Ilmenau.de
}

http://dx.doi.org/10.38028/esr.2020.04.0004

Received September 15, 2020. Revised October 14, 2020.

Accepted November 10, 2020. Available online February 01, 2021.

This is an open access article under a Creative Commons Attribution-NonCommercial 4.0 International License.

(C) 2020 ESI SB RAS and authors. All rights reserved.
The harmonic and supraharmonic distortion in electrical networks directly influence the power and energy losses in the electrical equipment. The harmonic and supraharmonic currents cause additional Joule heating of conductors and therefore increase the total power losses in these conductors.

The conductor resistance grows with a rise in the frequency and, therefore, relatively small harmonic or supraharmonic currents can cause notable power losses.

The studies presented in [1-3] show that additional power losses in LV cables and HV transmission lines caused by conventional current harmonics can be up to thirty percent of power losses at the fundamental frequency. The influence of supraharmonics on the additional power losses can be illustrated using the simplified assumption that the values of the line conductor resistances are proportional to the root of the frequency [2-5]:

$$
R_{s h}=R_{1} \sqrt{h},
$$

where $R_{s h}, R_{1}$ are resistance values at the supraharmonic frequency $f_{s h}$ and at the fundamental frequency $f_{1}$ respectively, $h=f_{s h} / f_{1}$ is the harmonic order.

Using the calculation formula for the Joule heating and taking into account (1), one can calculate the relative values of additional power losses caused by supraharmonics as follows:

$$
P_{L s h} / P_{L 1}=\left(I_{s h} / I_{1}\right)^{2} \sqrt{h},
$$

where $P_{L s h}, P_{L 1}$ are power losses caused by the supraharmonic current $I_{s h}$ at the frequency $f_{s h}$ and by the current $I_{1}$ at the fundamental frequency $f_{1}$, respectively.

The dependences (2) are presented in Figure 1 for some ratios of $I_{s h} / I_{1}$.

As seen from Figure 1, additional power losses caused by a supraharmonic can be, for example, ten percent of the power losses at the fundamental frequency and higher, and consequently, they cannot be neglected in the calculation of total power losses in electrical networks. The exact values of additional power losses depend on the supraharmonic frequency and the ratio of $I_{s h} / I_{1}$.

It is worth noting that (1) and (2) are valid mainly for overhead transmission lines. 


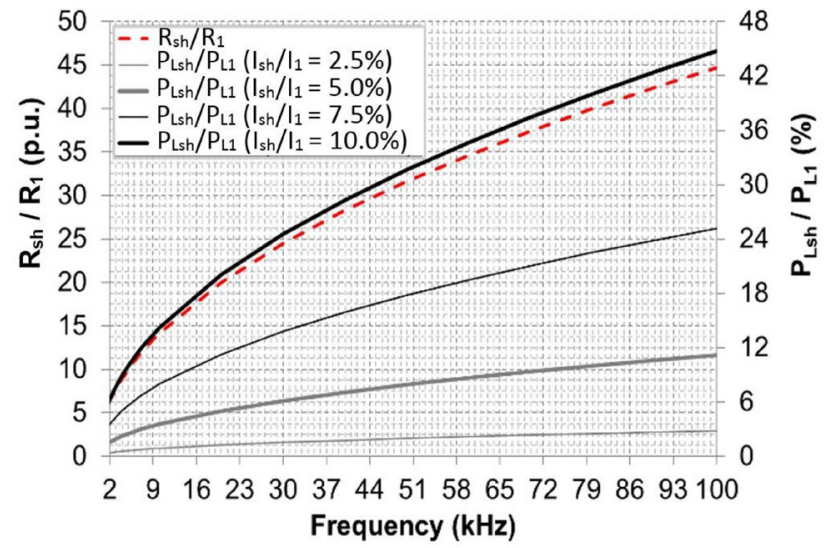

Fig. 1. Relative supraharmonic power losses (2) and the corresponding ratio of $R_{s h} / R_{1}$.
The estimation of power losses caused by supraharmonics in a cable feeder under real operating conditions is presented below. The estimation is based on the measurement results obtained in a real $\mathrm{MV} / \mathrm{LV}$ network in Germany [6].

\section{MEASUREMENTS OF SUPRAHARMONICS}

Results of the measurements carried out in the real $\mathrm{MV} / \mathrm{LV}$ electrical network presented in Figure 2 were used to estimate the effect of supraharmonics on power losses in MV cable feeders.

The network supplies power to different settlements with mainly residential and commercial loads and contains a lot of decentralized renewable energy generating units. The network contains a MV wind plant and several MV

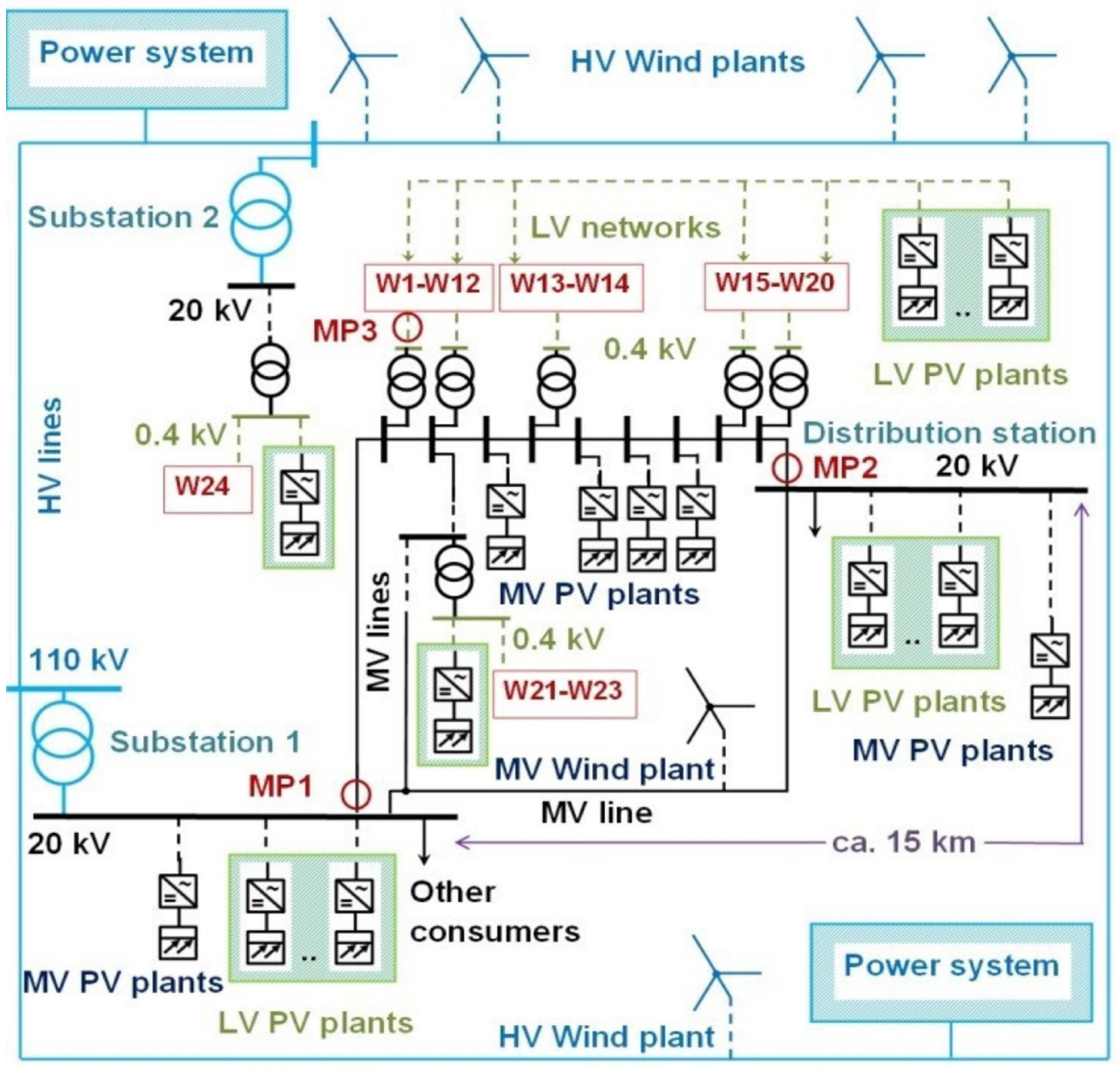

Fig. 2. MV/LV network under study and location of measuring devices. 


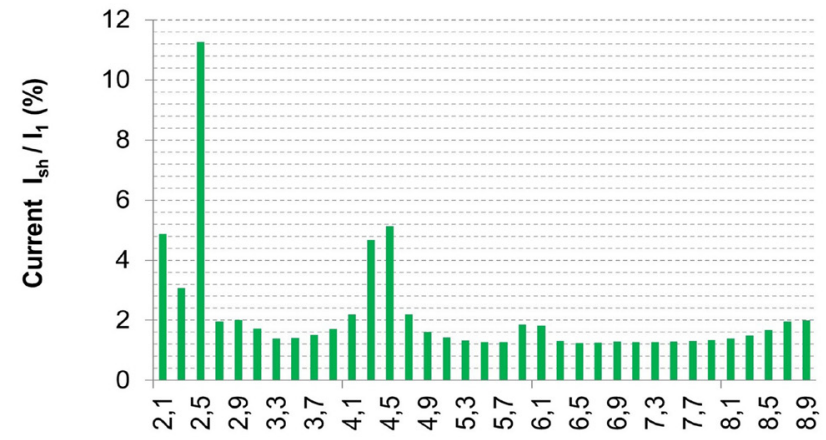

Frequency $(\mathbf{k H z})$

Fig. 3. Measured current spectrum, 95\% quantiles of 1 min. average values, $200 \mathrm{~Hz}$ groups, measurement time 24 h, MP2

PV plants distributed throughout the whole network and connected to the MV grid via dedicated transformers. Several powerful wind parks are located in the upstream HV network. Numerous LV PV power plants are connected to the downstream LV distribution networks together with other power electronic devices using high switching frequency power converters such as charging stations, consumer electronics, and others, via local area step-down MV / LV transformers.

Measurement devices SIRIUSi-HS [7] were located at the measurement points MP1 - MP3 and operated with a sampling rate of $100 \mathrm{kHz}$. Supraharmonic voltage and current $200 \mathrm{~Hz}$ groups (according to [8,9]) were recorded as $1 \mathrm{~min}$. average values over the measurement interval of 2 weeks.

Power quality (PQ) parameters were additionally measured at MP1 - MP3.

Instantaneous voltage values (a sampling rate of $10.240 \mathrm{kHz}$ ) and some PQ parameters as $1 \mathrm{sec}$ average values were recorded at the measurement points W1W24 (LV network) using measuring devices WeSense [10].

The effects of renewable energy sources on the supraharmonic distortion and the propagation of the supraharmonic distortion in the network under study were analyzed in $[6,11$, and 12].

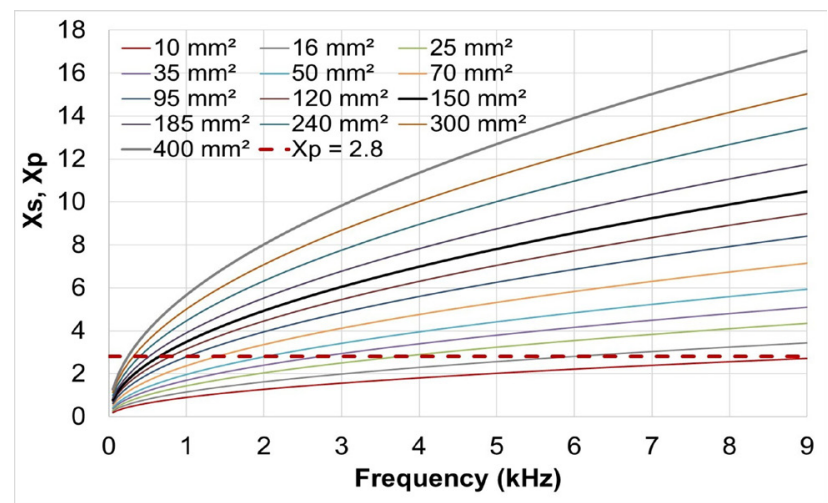

Fig. 5. Factors XS and Xp for cable solid aluminum conductors at the operating temperature of $20^{\circ} \mathrm{C}$.

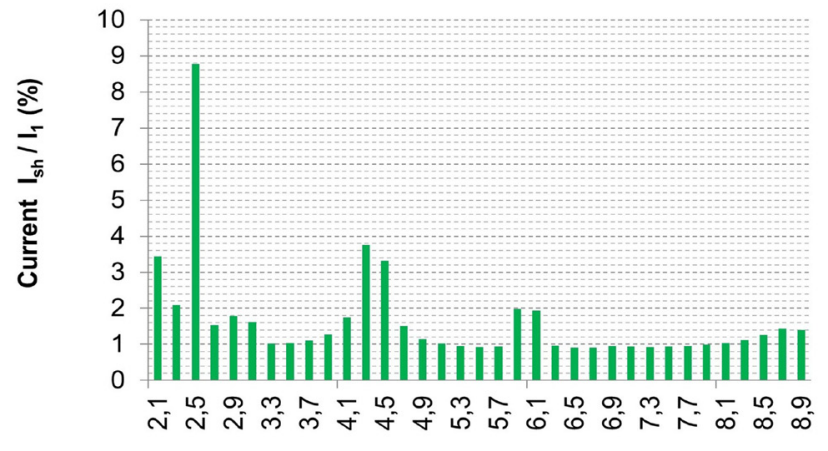

Frequency $(\mathbf{k H z})$

Fig. 4. Measured current spectrum, 95\% quantiles of 1 min. average values, $200 \mathrm{~Hz}$ groups, measurement time is one week, MP2

Power losses in the supraharmonic frequency range can be estimated using the supraharmonic currents measured in the MV feeder at the distribution station. This is the measurement point MP2 (Fig. 2).

Measuring device at MP2 was connected to the secondary winding of the current transformer via current clamps. Current clamps were certified for the measurements at a frequency up to $100 \mathrm{kHz}$.

The applicability of the standard MV current transformer to the measurements in the supraharmonic frequency range was assessed given the IEC Technical Report 61869-103 [13] and investigation results $[14,15]$.

Supraharmonic frequency range is divided into several subranges. The supraharmonics in the frequency subrange of 2 to $9 \mathrm{kHz}$ are the subject of special consideration in the IEC standards for compatibility levels for conducted disturbances and signaling in public medium-voltage [16] and low-voltage [17] power supply systems. Technical requirements for the operation of customer installations and their connection to the MV network in Germany contain admissible values for the supraharmonic currents in a frequency range of 2 to $9 \mathrm{kHz}$ [18]. Therefore, special attention should be paid to the transfer characteristics of MV current transformers in the frequency range of 2 to $9 \mathrm{kHz}$.

In [13], inductive current transformers are noted to be suitable for the use in the supraharmonic frequency range of 2 to $9 \mathrm{kHz}$. Given the measured frequency dependences

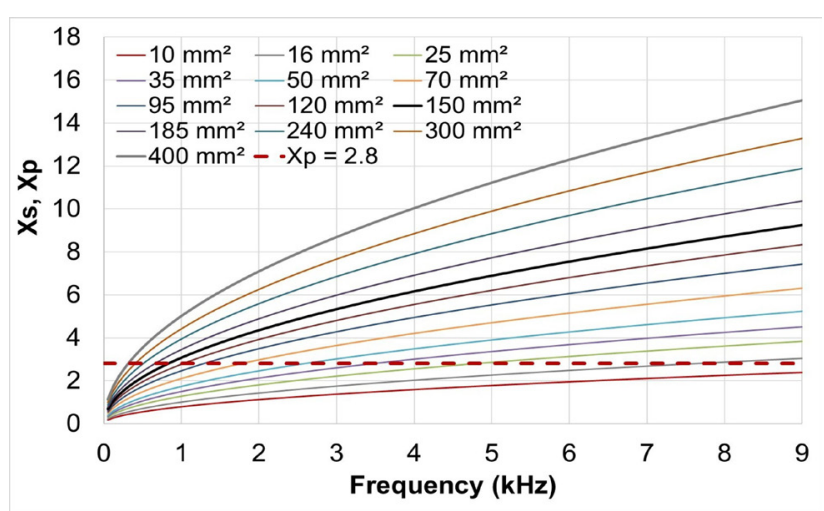

Fig. 6. Factors $X_{S}$ and $X_{p}$ for cable solid aluminum conductors at the operating temperature of $90^{\circ} \mathrm{C}$ 


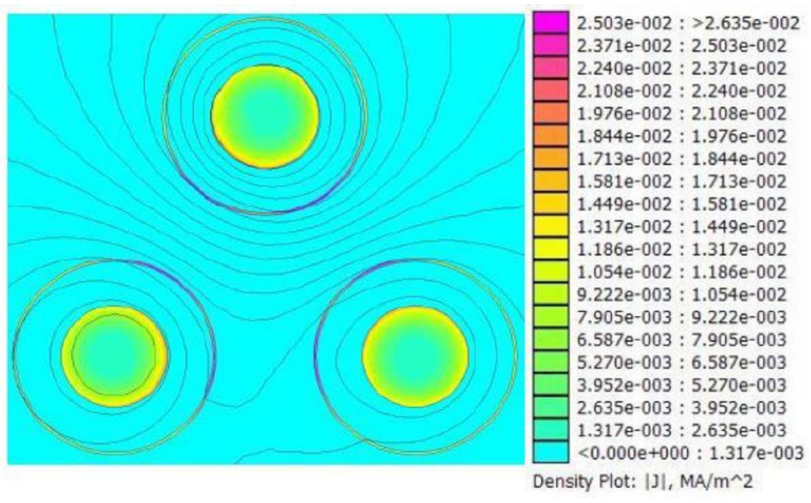

Fig. 7. Current density distribution in the aluminum conductors and copper screens of the MV cable 3x1x150/25 caused by the supraharmonic current $0.54 \mathrm{~A}$ (r.m.s. value) at the frequency of $2.5 \mathrm{kHz}$

for ratio and phase errors of the MV current transformers presented in [14] and [15], one can conclude that the supraharmonic currents can be measured with the standard MV current transformers with sufficient accuracy (an error amplitude of up to several percent) for the simplified estimation of power losses in the frequency range of 2 to 9 $\mathrm{kHz}$.

Examples of measured current spectra in the MV feeder (MV distribution station, measurement point MP2) are presented in Figs. 3 and 4.

As seen in Figures 3 and 4, the current spectra are characterized by the domination of the supraharmonic components of $2.5 \mathrm{kHz}$. In $[6,12]$, the authors show that these components in the considered network are mainly caused by the operation of the MV wind plant.

The components of 5.9 and $6.1 \mathrm{kHz}$ are caused by the daily operation of solar plants, the components of 4.3 and $4.5 \mathrm{kHz}$ result from the changes at the connection points of operating units.

The measurement point MP2 is the connection point of the MV $3 \times 1 \times 150 / 25$ cable to the busbar of the distribution station. This MV cable connection contains three single-core cables with $150 \mathrm{~mm}^{2}$ aluminum conductors and $25 \mathrm{~mm}^{2}$ copper screens for each of them.

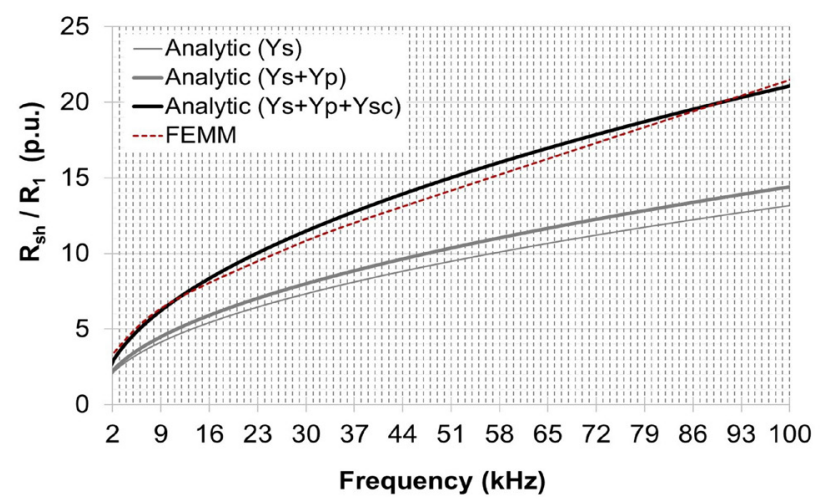

Fig. 9. Analytically and numerically (using the FEMM software) determined frequency dependences Rsh / R1 for the MV 3x1x150/25 cable in a supraharmonic frequency range

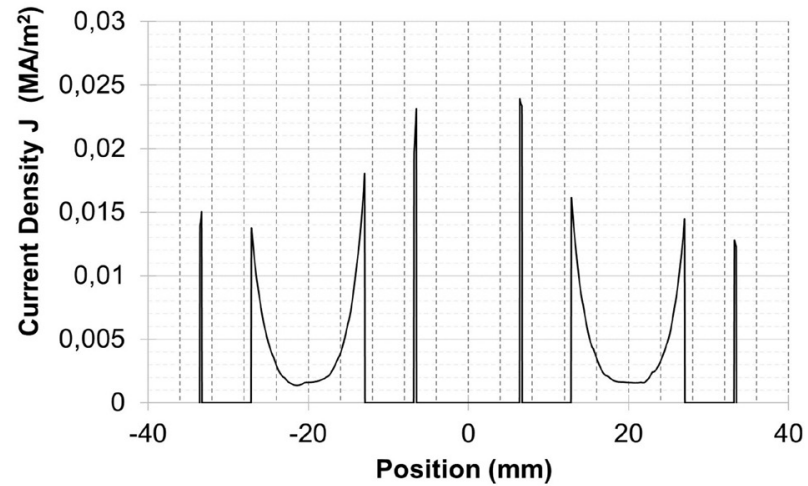

Fig. 8. Current density distribution $J$ along the straight line crossing the centers of two neighboring conductors and screens of the MV 3x1x150/25 cable caused by the supraharmonic current 0.54 A (r.m.s. value) at the frequency $2.5 \mathrm{kHz}$

Determination of the frequency dependences for the cable resistances is considered below.

\section{DETERMINATION OF THE FREQUENCY DEPENDENCES FOR THE CABLE RESISTANCES}

\section{A. Analytic calculation method}

Calculation of AC resistance of a conductor is part of the IEC standard [19]. The standard states that the AC cable conductor resistance depends on the skin and the proximity effects. The following formula can be used:

$$
R_{s h}=R_{D C}^{\prime}\left(1+Y_{S}+Y_{P}\right)
$$

where $R_{s h}$ is a conductor resistance at the supraharmonic frequency $f_{s h}, R_{D C}^{\prime}$ is a DC resistance of conductor at the maximum operating temperature $\theta, Y_{S}$ is a skin effect factor, and $Y_{p}$ is a proximity effect factor.

The values of $Y_{S}$ and $Y_{p}$ depend on the values of the factors $X_{S}$ and $X_{p}$ as follows:

and

$$
X_{S}^{2}=\left(1 / R_{D C}^{\prime}\right) 8 \pi f_{s h} 10^{-7} k_{S}
$$

$$
X_{P}^{2}=\left(1 / R_{D C}^{\prime}\right) 8 \pi f_{s h} 10^{-7} k_{P},
$$

where $k_{S}, k_{p}$ are coefficients, e.g. $k_{S}=1$ and $k_{p}=1$ for cables with solid copper or aluminum conductors [19].

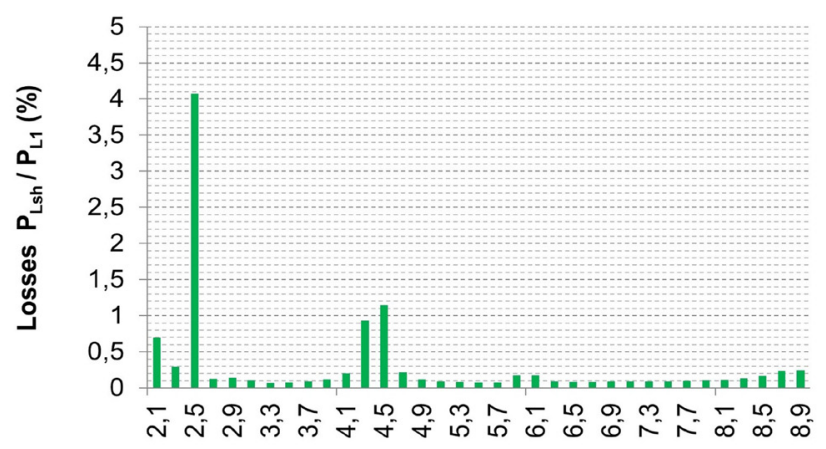

Frequency $(\mathbf{k H z})$

Fig. 10. Relative power losses in a $20 \mathrm{kV}$ cable feeder, 95\% quantiles of 1 min. values, $200 \mathrm{~Hz}$ groups, measurement time is $24 \mathrm{~h}, \mathrm{MP} 2$ 


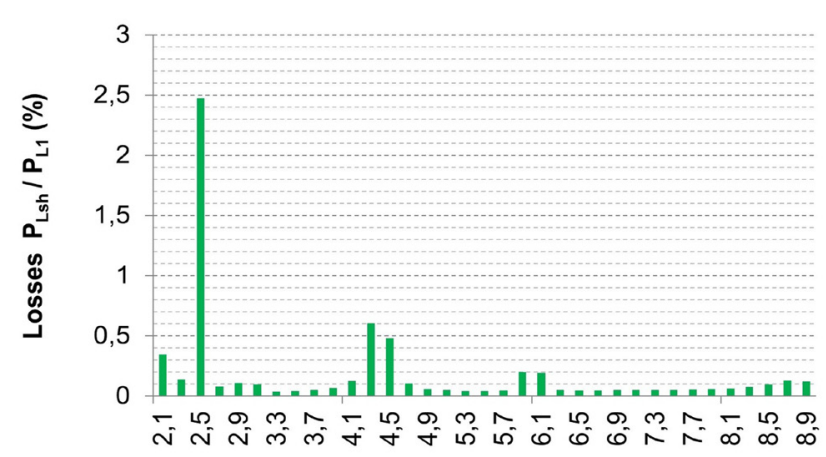

Frequency $(\mathbf{k H z})$

Fig. 11. Relative power losses in a $20 \mathrm{kV}$ cable feeder, 95\% quantiles of $1 \mathrm{~min}$. values, $200 \mathrm{~Hz}$ groups, measurement time is one week, MP2

The skin effect factor $Y_{S}$ is given by the following equations [19]:

for $0<X_{S} \leq 2.8$

$$
Y_{S}=X_{S}^{4} /\left(192+0.8 X_{S}^{4}\right),
$$

for $2.8<X_{S} \leq 3.8$

$$
Y_{S}=-0.136-0.0177 X_{S}+0.056 X_{S}^{2}
$$

for $3.8<X_{S}$

$$
Y_{S}=0.354 X_{S}-0.733
$$

The proximity effect factor $Y_{p}$, according to [19], can be calculated only if factor $X_{p}$ does not exceed 2.8 .

As seen from (4) and (5), on the assumption that $k_{S}=k_{p}=1$, for the cables with solid conductors $X_{p}=X_{S}[19]$.

The values of $X_{p}$ and $X_{S}$ calculated according to (4) and (5) for the cables with solid aluminum conductors are presented in Figures 5 and 6. The values $R_{D C}^{\prime}$ in (4) and (5) were determined for each cross-sectional area according to [20], based on the standard formula [19]:

$$
R_{D C}^{\prime}=R_{D C}\left(1+\alpha_{20}(\theta-20)\right),
$$

where $R_{D C}$ is a DC resistance of conductor at $20^{\circ} \mathrm{C}, R_{D C}^{\prime}$ is a $\mathrm{DC}$ resistance of conductor at the maximum operating temperature $\theta, \alpha_{20}$ is the constant mass temperature coefficient at $20^{\circ} \mathrm{C}$ per Kelvin.

The calculation results for the operating temperature of $20^{\circ} \mathrm{C}$ are presented in Figure 5, the calculation results for the maximum operating temperature of $90^{\circ} \mathrm{C}$ are presented in Figure 6.

It can be clearly seen from Figures 5 and 6 that the cable solid aluminum conductors with the standard cross-sectional areas starting with $16 \mathrm{~mm}^{2}$ and larger are characterized by the values of $X_{p}$ higher than 2.8 at least at some frequencies in the frequency range of 2 to $9 \mathrm{kHz}$.

Cable solid aluminum conductors with the standard cross-sections of $70 \mathrm{~mm}^{2}$ and larger are characterized by the values of $X_{p}$ higher than 2.8 in the entire frequency range of 2 to $9 \mathrm{kHz}$.

This means that the method for the analytic calculation of frequency-dependent cable resistances [19] is not applicable to the calculation of resistances at supraharmonic frequencies in many practical cases due to the constraints on the combination of parameters considered in [19].

A more common analytic method for the calculation of frequency-dependent cable resistances is presented in [21].

The analytic method for the calculation of the skin effect factor $Y_{S}$ in [21] is identical with the method [19] described above.

The following formula is suggested in [21] for the calculation of the proximity effect factor $Y_{p}$ for three single-core cables:

$$
Y_{P}=m y^{2} G_{P} /\left(2-5 y^{2} H_{P} / 12\right),
$$

where $m=3$ for three single-core circular cables; $y$ is a spacing ratio; $G_{p}, H_{p}$ are coefficients.

The coefficients $G_{p}, H_{p}$ are given by the following equations [21]:

for $0<X_{p} \leq 2.8$

$$
G_{P}=11 X_{P}^{4} /\left(704+20 X_{P}^{4}\right),
$$

for $2.8<X_{p} \leq 3.8$

$$
G_{P}=-0.08 X_{P}^{2}+0.72 X_{P}-1.04,
$$

for $3.8<X_{p}$

for $0<X_{p} \leq 2.8$

$$
G_{P}=X_{P} /(4 \sqrt{2})-1 / 8,
$$

$$
H_{P}=(1 / 3)\left(1+0.0283 X_{P}^{4}\right) /\left(1+0.0042 X_{P}^{4}\right),
$$

for $2.8<X_{p} \leq 3.8$

$$
H_{P}=0.0384 X_{P}^{2}+0.119 X_{P}+0.095,
$$

for $3.8<X_{p}$

$$
H_{P}=\frac{2 X_{P}-4.69}{X_{P}-1.16} .
$$

The spacing ratio $y=d_{c} / s$, where $s$ is the spacing between conductor axes, $d_{c}$ is the diameter of an equivalent circular conductor.

The construction of the MV $3 \times 1 \times 150 / 25$ cable suggests that each cable conductor is surrounded by a cable screen.

The AC currents flowing in the cable conductors cause induced currents in the screens surrounding the cable and therefore cause some additional power losses in the screens. This means an increase in the equivalent cable resistances at the frequencies of the $\mathrm{AC}$ currents (fundamental frequency, harmonic and supraharmonic frequencies).

Therefore, formula (3) can be extended to consider the influence of power losses in cable screens on the conductor resistance as follows:

$$
R_{s h}=R_{D C}^{\prime}\left(1+Y_{S}+Y_{P}+Y_{S C}\right),
$$

where $Y_{S C}$ is the screen losses factor.

It is worth noting that each cable containing axial conductor surrounded by a cable screen can be considered as a pipe-type cable. Taking into account the relation given in [22] to consider the increase in losses in the phase 
conductors due to the proximity of the pipe, we can use the following formula for the screen loss factor:

$$
Y_{S C}=0.5\left(Y_{S}+Y_{P}\right) \text {. }
$$

These considerations are in compliance with the recommendation [19] for the calculation of AC cable conductor resistances for pipe-type cables. The following formula can be used according to [19]:

$$
R_{s h}=R_{D C}^{\prime}\left(1+1.5\left(Y_{S}+Y_{P}\right)\right) \text {. }
$$

Using (4) - (16), (19), one can analyticly determine the values of the conductor resistances at the supraharmonic frequencies $R_{s h}$ and the relations $R_{s h} / R_{1}$ for the $\mathrm{MV}$ $3 \times 1 \times 150 / 25$ cable under study.

\section{B. Numerical calculation method}

The use of the finite element method (FEM) is a popular approach to the numerical calculation of frequency dependences for cable resistances.

The advantages of this method are the exact modeling of the cable geometry, the simulation of properties of the materials used in the cable construction, and the simultaneous consideration of all influencing effects named above: the skin effect, the proximity effect, the influence of cable screens and all other metallic elements (for example, cable armor, pipes, ducts, trays, and others), which can affect the AC resistances of the cable conductors.

The FEM approach makes it possible to calculate the current density distribution in all parts of the simulated cable construction. Taking into account the cable geometry and the electrical conductivities of simulated constructive materials, one can determine power losses in all elements of the cable construction.

The equivalent $\mathrm{AC}$ resistance of the cable conductor $R_{s h}$ at the supraharmonic frequency $f_{s h}$ can be calculated considering the following formula:

$$
P_{L s h}=I_{s h}^{2} R_{s h},
$$

where $P_{L s h}$ is the average value of total power losses caused by the simulated supraharmonic conductor current $I_{s h}$ at the frequency $f_{s h}$ in each phase of the three-phase cable system.

In respect to the considered MV 3x1 x150/25 cable construction:

$$
P_{L s h}=P_{L s h \text { cond }}+P_{L s h s c},
$$

where PLsh cond is an average value of power losses in each phase conductor and PLsh sc is an average value of power losses in each conductor screen. The frequency dependence for the equivalent $\mathrm{AC}$ cable resistance can be determined through a series of simulations at different frequencies. The frequency dependence can be determined from the following relations:

$$
P_{L s h} / P_{L 1}=I_{s h}^{2} R_{s h} /\left(I_{1}^{2} R_{1}\right) .
$$

The values $I_{s h}$ and $I_{1}$ can be taken, for example, from the measurement results.
Taking into account the FEM simulation of the heating processes, the cable operating temperature can be determined and the temperature effect on the electrical conductivity can be considered additionally [23-25].

To simplify the calculations, it is sufficient to assume that $I_{s h}=I_{1}$. In this case, it is enough to calculate the power losses (21) for each frequency under consideration. Formula (22) will be simplified as follows:

$$
P_{L s h} / P_{L 1}=R_{s h} / R_{1} .
$$

The frequency dependence for the equivalent $\mathrm{AC}$ cable resistance can be determined using (23).

It is noteworthy that the electrical conductivity of the cable insulation is very small in comparison with the conductivities of cable conductors and metallic screens. Therefore, it is sufficient to simulate only metallic parts of the cable configuration to simplify the estimation of power losses (20) and to calculate the equivalent $\mathrm{AC}$ resistance of the cable conductor.

Figure 7 presents a simulation example for the MV $3 \times 1 \times 150 / 25$ cable. The trefoil formation with a spacing between conductor axes of $40 \mathrm{~mm}$ was simulated. Both aluminum conductors and copper screens were simplified and represented as solid objects.

The simulation was carried out using the FEMM software package [26].

The calculated current density distribution $J$ in the aluminum cable conductors and in the copper cable screens is shown in Figure 7. The supraharmonic phase conductor currents of $I_{s h}=0.54 \mathrm{~A}$ (r.m.s. value) at the frequency $f_{s h}=2.5 \mathrm{kHz}$ were simulated as the origin of the magnetic field in the cable. This value of the conductor current was measured as a 1 min value during the measurement campaign at the measurement point MP2.

The conductor currents were simulated as a threephase set of balanced phasors with a $120^{\circ}$ difference in phase angles between two neighboring phasors.

Figure 8 presents the line plot of the current density for the straight-line contour crossing the centers of two neighboring conductors with screens. The coordinates of centers in the "position" axis are $-20 \mathrm{~mm}$ and $+20 \mathrm{~mm}$, respectively. The Figure also indicates skin effect, proximity effect, and influence of cable screens. Calculated frequency dependences for the MV cable under study

The analyticly calculated frequency dependences

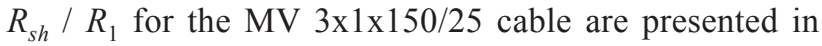
Figure 9. These are the frequency dependence calculated taking into account only the skin effect (parameter $Y_{S}$ ), the frequency dependence calculated taking into account both the skin effect and the proximity effect (parameters $Y_{S}$ and $Y_{p}$ ), the frequency dependence calculated taking into account the skin effect, the proximity effect, and the influence of power losses in the conductor screens (parameters $Y_{S}, Y_{p}, Y_{S C}$ ). 
The frequency dependence $R_{s h} / R_{1}$ determined with the FEMM software package for numerical calculations is presented for comparison in Figure 9.

The corresponding reference values $R_{s h} / R_{1}=1$ were calculated for each presented dependence, in terms of the fundamental frequency of $50 \mathrm{~Hz}$.

Figure 9 shows that the skin effect is the dominating factor in the increase in the equivalent $\mathrm{AC}$ resistance of the cable conductor $R_{s h}$ with a rise in the frequency in the supraharmonic frequency range.

As seen in Figure 9, the influence of power losses in conductor screens on the increase in the equivalent $\mathrm{AC}$ resistance of the cable conductor $R_{s h}$ with a rise in the frequency is much higher in comparison with the influence of the proximity effect for the MV cable under study.

Based on the comparison of the analyticly and numerically calculated frequency dependences presented in Figure 9, we can conclude that the analytic formula (17), parameterized according to (18), or the direct formula (19) are most suitable for the analytic characterization of the increase in the equivalent $\mathrm{AC}$ resistance of the cable conductor $R_{s h}$ when the frequency for the MV cable under study goes up in the supraharmonic frequency range of 2 to $100 \mathrm{kHz}$.

Given the frequency dependence (1) presented graphically in Figure 1, it can be concluded that the frequency dependences calculated by (19) or using FEMM simulations for the MV cable under study are characterized by lower values of the relations $R_{s h} / R_{1}$ than the square root of the harmonic order $h=f_{s h} / f_{1}$.

For the supraharmonic frequency range of 2 to $9 \mathrm{kHz}$, the following formula can be suggested for a simplified representation of the frequency dependence of $R_{s h} / R_{1}$ for the MV cable under study:

$$
R_{s h} / R_{1} \approx 0.48 \sqrt{h} \text {. }
$$

Deviations of (24) from the FEMM calculation results do not exceed several percent points in the range of 2 to $9 \mathrm{kHz}$.

\section{USE OF MEASUREMENT RESULTS FOR THE SUPRAHARMONIC POWER LOSS ESTIMATION}

The supraharmonic power losses in the MV cable under study can be determined using (22). For clarity, (22) can be represented similar to (2) and rewritten as follows:

$$
P_{L s h} / P_{L 1}=\left(I_{s h}^{2} / I_{1}^{2}\right) R_{s h} / R_{1} .
$$

Taking into consideration the calculated frequency dependences $R_{s h} / R_{1}$ for the MV cable under study and the measurement results for the time series $I_{s h} / I_{1}$ obtained during the measurement campaign, we can determine the time series for the relative values of supraharmonic power losses $P_{L s h} / P_{L 1}$.

Figures 10 and 11 show the supraharmonic spectra of relative power losses in the frequency range of 2 to 9 $\mathrm{kHz}$ in the $20 \mathrm{kV}$ cable feeder under study determined according to (25) for the measurement interval of 24 hours (Figure 10) and for the measurement interval of one week (Figure 11).

The spectra in Figures 10 and 11 are presented for the supraharmonic groups of $200 \mathrm{~Hz}$ in accordance with the obtained measurement results for the supraharmonic currents. For the centered frequencies of each group, the frequency dependences $R_{s h} / R_{1}$ calculated according to (19) were taken into consideration.

It is worth noting that the sum of average values of 1 min. relative harmonic power losses (harmonics 2 to 40 ) for this day of measurement considered in Figure 10 is $24.4 \%$, the sum of average values of 1 min relative supraharmonic power losses in the range of 2 to $9 \mathrm{kHz}$ presented in Figure 10 is $2.4 \%$. The sum of average values of $1 \mathrm{~min}$ relative harmonic power losses for the whole measurement week considered in Figure 11 is $12.9 \%$, the sum of average values of 1 min relative supraharmonic power losses in the range of 2 to $9 \mathrm{kHz}$ presented in Figure 11 is $1.6 \%$.

This means that there are supraharmonic power losses in modern distribution networks and they can reach the values of several percent of power losses at the fundamental frequency. The analysis shows that the supraharmonic power losses can exceed ten percent of power losses caused by conventional current harmonics in the MV cable under study. Therefore, it can be recommended to consider supraharmonic power losses to correctly estimate the total power losses in modern distribution networks.

\section{CONCLUSION}

The paper has considered a method of estimating the supraharmonic power losses, which combines the analytic determination of $\mathrm{AC}$ cable resistances at the supraharmonic frequencies and the use of measurements of supraharmonic currents in a real MV/LV cable network.

The results of the analytic calculations of the AC cable resistances at supraharmonic frequencies have been verified and made more precise using the FEMM simulations for a MV cable chosen for the investigation. We have proposed a simplified formula for the estimation of the increase in the AC cable resistances with respect to the cable resistance at the fundamental frequency of the MV cable under study for the frequency range of 2 to $9 \mathrm{kHz}$.

The findings indicate that the estimation of the supraharmonic power losses in MV networks in the frequency range of 2 to $9 \mathrm{kHz}$ can be simplified using the measurement results obtained by conventional current measuring instruments.

The study has shown that supraharmonic power losses in the considered MV cable operating in a real network can reach the values of several percent of power losses at the fundamental frequency and can exceed ten percent of the power losses caused by conventional current harmonics.

It can be recommended to consider supraharmonic power losses to correctly estimate the total power losses in networks with high presence of power electronic devices. 


\section{REFERENCES}

[1] L. Topolski, J. Warecki, Z. Hanzelka, "Calculating Power Losses In LV Cables Loaded With Nonsinusoidal Currents," in Proc. CPEE, Banska Stiavnica, Slovakia, 2018, pp. 25-28.

[2] N. Kharlov, V. Borovikov, V. Litvak, A. Pogonin, V. Melnikov, "Energy survey of non-sinusoidal operating states of multiconductor power transmission lines," Electrichestvo (Electricity), 2011, no.12, pp. 12-15.

[3] N. Kharlov, V. Borovikov, "Results of a survey of operating states of distribution electrical networks of Siberia and southern Russia," in Proc. Energy-21: Sustainable Development \& Smart Management, Irkutsk, Russia, 2015, pp. 183-188.

[4] J. Zhu, E. Bećirović, J. Milanović, "The Effect of Network Modelling on Harmonic Propagation Studies in Power Electronics Rich Transmission Networks," in Proc. APSCOM, Hong Kong, China, 2018, pp. 1-6.

[5] G. J. Wakileh, Power Systems Harmonics. Berlin Heidelberg, Germany: Springer Verlag, 2001, p. 221.

[6] A. Novitskiy, S. Schlegel, D. Westermann, "Measurements and Analysis of Supraharmonic Influences in a MV/LV Network Containing Renewable Energy Sources," in Proc. $P Q$ \& SEEM, Kärdla, Estonia, 2019, pp. 1-6.

[7] SIRIUS ${ }^{\circledR}$ Technical Reference Manual, DEWESoft $\mathrm{GmbH}$, Kumberg, Austria, 2015.

[8] Electromagnetic compatibility (EMC) - Part 4-7: Testing and measurement techniques - General guide on harmonics and interharmonics measurements and instrumentation, for power supply systems and equipment connected thereto, IEC 61000-4-7, 2009.

[9] Electromagnetic compatibility (EMC) - Part 4-30: Testing and measurement techniques - Power quality measurement methods, IEC 61000-4-30, 2015.

[10] C. Rüster, F. Haussel, T. Hühn, N. El Sayed, "VEREDELE-FACDS Field Trial: Wide Area Power Quality Assessment with IOT Sensors and CloudBased Analytics," in Proc. ETG Congress, Bonn, Germany, 2017, pp. 1-5.

[11] A. Novitskiy, S. Schlegel, D. Westermann, “Analysis of Supraharmonic Propagation in a MV Electrical Network," in Proc. EPE, Brno, Czech Republic, 2018, pp. 1-6.

[12] A. Novitskiy, P. Tikhonov, T. Jiang, S. Schlegel, T. Hühn, N. El Sayed, C. Rüster, D. Westermann. "Influence of Renewable Energy Sources on Supraharmonic Distortion in Modern MV/LV Distribution Networks," in Proc. PQM, Moscow, Russia, 2018, pp. 142-147.

[13] Instrument Transformers - The use of instrument transformers for power quality measurement, IEC/TR 61869-103, 2012.

[14] K. Kunde, H. Däumling, R. Huth, H.-W. Schlierf, J. Schmid, "Frequency Response of Instrument Transformers in the kHz range," etz, v. 6, pp. 1-4, 2012.
[15] M. Redfern, S. Terry, F. Robinson, Z. Bo. "A Laboratory Investigation into the use of $\mathrm{MV}$ Current Transformers for Transient Based Protection," in Proc. IPST, New Orleans, LA, USA, 2003, pp. 1-6.

[16] Electromagnetic compatibility (EMC) - Part 2-12: Environment - Compatibility levels for lowfrequency conducted disturbances and signaling in public medium-voltage power supply systems. IEC 61000-2-12, 2003.

[17] Electromagnetic compatibility (EMC) - Part 2-2: Environment - Compatibility levels for lowfrequency conducted disturbances and signaling in public low-voltage power supply systems, IEC 61000-2-2, 2018.

[18] Technical Requirements for the connection and operation of customer installations to the medium voltage network (TAR medium voltage), VDEAR-N-4110. 2018.

[19] Electric cables - Calculation of the current rating Part 1-1: Current rating equations (100\% load factor) and calculation of losses - General. Ed. 2.1, IEC 60287-1-1, 2014.

[20] Conductors of insulated cables. Ed. 3.0, IEC 60228, 2004.

[21] Y. Du and J. Burnett, "Experimental Investigation into Harmonic Impedance of Low-Voltage Cables," IEE Proceedings - Generation, Transmission and Distribution, vol. 147, issue 6, pp. 322-328, Dec. 2000.

[22] J. Palmer, R. Degeneff, T. McKernan, T. Halleran, "Pipe-Type Cable Ampacities in the Presence of Harmonics," IEEE Transactions on Power Delivery, vol. 8, No. 4, pp. 1689-1695, Oct. 1993.

[23] W. Frelin, L. Berthet, Y. Brument, M. Petit, G. Perujo, J. Vannier. "Thermal Behavior of LV Cables in Presence of Harmonic Currents," in Proc, ISEF, Arras, France, 2009, pp. 1-8.

[24] S. Dubitsky, G. Greshnyakov, N. Korovkin. "Refinement of Underground Power Cable Ampacity by Multiphysics FEA Simulation," Int. Journal of Energy, vol. 9, pp. 12-19, 2015.

[25] Electric Cables - Calculations for Current RatingsFinite Element Method, IEC/TR 62095, 2003.

[26] D. Meeker, "Finite Element Method Magnetics, User's Manual," Version. 4.2, May 2020. 


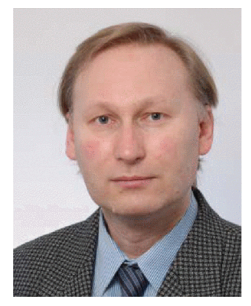

Alexander Novitskiy received his $\mathrm{Ph} . \mathrm{D}$. degree in electrical engineering from St. Petersburg State Polytechnic University in 1993. In 1994-1995, he worked as a Researcher at Tianjin University of Technology, China. Since 1996, he has been a Researcher at Ilmenau University of Technology, Germany. In 2006, he received his Dr.-Ing. habil. (D.Sc.) degree from that University. Since 2007, he has been a Senior Scientist in the Power Systems Group, Department of Electrical Engineering and Information Technology. His research interests include power quality, electromagnetic compatibility and interferences, simulation and analysis of steady states and transients in electric power systems.

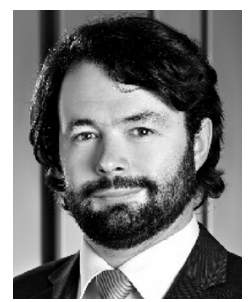

Steffen Schlegel received his Ph.D. degree in electrical engineering from Ilmenau University of Technology, Ilmenau, Germany, in 2015. He is Chief Engineer and Head of the research team "Vertical System Operation" at Power Systems Group. A special focus of his research is on system integration of transmission and distribution grid operation, new power system operation methodologies with an active interface between distribution and transmission grid operators.

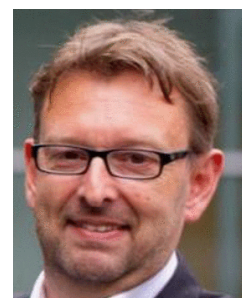

Dirk Westermann received the Diploma in electrical engineering and the Ph.D. degree from the University of Dortmund, Dortmund, Germany, in 1992 and 1997, respectively. In 1997, he joined the ABB Switzerland Ltd., where he held several positions in R\&D and Technology Management. He became a Full Professor of power systems at Ilmenau University of Technology in 2005. Since 2018, he has been the Director of the Thuringian Energy Research Institute.

His research interests include design, control and operation of power systems. $\mathrm{He}$ is an IEEE Senior Member, an active member of CIGRE and IEC working groups. 